\title{
Advancing classical and quantum communication systems with machine learning
}

Zibar, Darko; Moura, Uiara Celine de; Chin, Hou-Man; Brusin, Ann M. Rosa; Jain, Nitin; Da Ros, Francesco; Kleis, Sebastian; Schaeffer, C. ; Gehring, Tobias; Andersen, Ulrik Lund

Total number of authors:

11

Published in:

Optical Fiber Communication Conference 2020

Link to article, DOI:

10.1364/OFC.2020.W1K.1

Publication date:

2020

Document Version

Peer reviewed version

Link back to DTU Orbit

Citation (APA):

Zibar, D., Moura, U. C. D., Chin, H-M., Brusin, A. M. R., Jain, N., Da Ros, F., Kleis, S., Schaeffer, C., Gehring, T., Andersen, U. L., \& Carena, A. (2020). Advancing classical and quantum communication systems with machine learning. In Optical Fiber Communication Conference 2020 [W1K.1] IEEE.

https://doi.org/10.1364/OFC.2020.W1K.1

\section{General rights}

Copyright and moral rights for the publications made accessible in the public portal are retained by the authors and/or other copyright owners and it is a condition of accessing publications that users recognise and abide by the legal requirements associated with these rights.

- Users may download and print one copy of any publication from the public portal for the purpose of private study or research.

- You may not further distribute the material or use it for any profit-making activity or commercial gain

- You may freely distribute the URL identifying the publication in the public portal 


\title{
Advancing classical and quantum communication systems with machine learning
}

\author{
D. Zibar ${ }^{1}$, U. C. Moura ${ }^{1}$, H. M. Chin ${ }^{2}$, A. M. Rosa Brusin ${ }^{3}$, N. Jain ${ }^{2}$, F. Da Ros ${ }^{1}$, \\ S. Kleis ${ }^{4}$, C. Schaeffer ${ }^{4}$, T. Gehring ${ }^{2}$, U. L. Andersen ${ }^{2}$ and A. Carena ${ }^{3}$ \\ 1. DTU Fotonik, Technical University of Denmark, DK-2800, Kgs. Lyngby, Denmark \\ 2. Center for Macroscopic Quantum States, Department of Physics, Technical University of Denmark, DK-2800 \\ Kgs. Lyngby, Denmark \\ 3. Dipartimento di Elettronica e Telecomunicazioni, Politecnico di Torino, Corso Duca degli Abruzzi, 24 - 10129, \\ Torino, Italy \\ 4. Faculty of Electrical Engineering, Helmut Schmidt University, 22008 Hamburg, Germany \\ dazi@fotonik.dtu.dk
}

\begin{abstract}
A perspective on how machine learning can aid the next-generation of classical and quantum optical communication systems is given. We focus on the design of Raman amplifiers and phase tracking at the quantum limit. () 2020 The Author(s)
\end{abstract}

OCIS codes: (060.1660) Coherent communications, (060.2360) Fiber optics links and subsystems

\section{Introduction}

According to the recent data traffic predictions, current optical communication systems, operating in the C-band only, will not be able to satisfy future data rate demands [1]. A viable and long-term solution would be to employ systems operating in multiple bands $(\mathrm{O}+\mathrm{E}+\mathrm{S}+\mathrm{C}+\mathrm{L})[2]$ and make usage of the spatial division multiplexing (SDM) (multi-core and multi-mode) [1]. Designing optimal signaling and detection schemes, for such systems, will be challenging due to the high system complexity. Moreover, optimizing amplification schemes would require fast tuning of a very large number of parameters as arbitrary gain profiles will be highly desired. Finally, performing system optimization in terms of channel power and bandwidth allocation, as well as modulation format selection, will become difficult using standard tools that rely on analytical or semi-analytical models.

Another important aspect is the requirement on providing secure communication, and this is where quantum technologies come into a play [3]. Quantum key distribution (QKD) technology is a key component in securing future communication systems. There are two main flavours of QKD, discrete variable and continuous variable, (DV-QKD) and (CV-QKD), respectively [3]. The advantage of CV-QKD is that standard telecom components can be used, making the systems more affordable. Moreover, CV-QKD relies on coherent detection and digital signal processing (DSP) techniques, resembling the classical coherent communication systems to a large extent. This implies that the know-how obtained from the classical coherent communication systems over the last ten years can be reused. There are however, also, significant differences between the classical and quantum optical communication systems.

In classical optical communication, it is common to amplify the signals as they propagate through the optical fiber. The optical signal power at the receiver can therefore be restored and the mean photon number per bit can easily exceed 1000, translating into moderate values of signal-to-noise-ratio (SNR). In quantum communication, the SNR is low because most quantum protocols work with less than one photon per symbol in average and also because optical amplification is not allowed as it would destroy a quantum state. Therefore, for applications in quantum communication, and optical communication where optical amplifiers cannot be used (e.g. in space communication), the systems are quantum noise limited. Moreover, the requirements on the level of accuracy of optical phase noise tracking for CV-QKD are significantly more stringent compared to classical systems [9]. This implies that quantum-noise limited performance of optical phase noise tracking needs to be achieved from optical signals having significantly lower power levels compared to the signals in classical optical communication.

The field of machine learning (ML) can provide useful tools to address the aforementioned challenges. This is because ML techniques excel at: 1) learning highly-complex input-output mappings which allows for system optimization [4-6], 2) learning signaling and detection schemes for complex channels or for channels where analytical models are not available [7,8], and 3) performing ultra-sensitive signal detection $[9,10]$. In this paper, it will be demonstrated how multi-layer neural networks, which are one of the most popular machine learning technique nowadays, can enable an inverse system design (ISD). For the ISD, we are given a target (desired) output and the task is to determine the corresponding set of inputs. The ISD is highly relevant for the design of ultra-wide band Raman and SDM amplifiers where a large number of pumps needs to be adjusted to produce a 


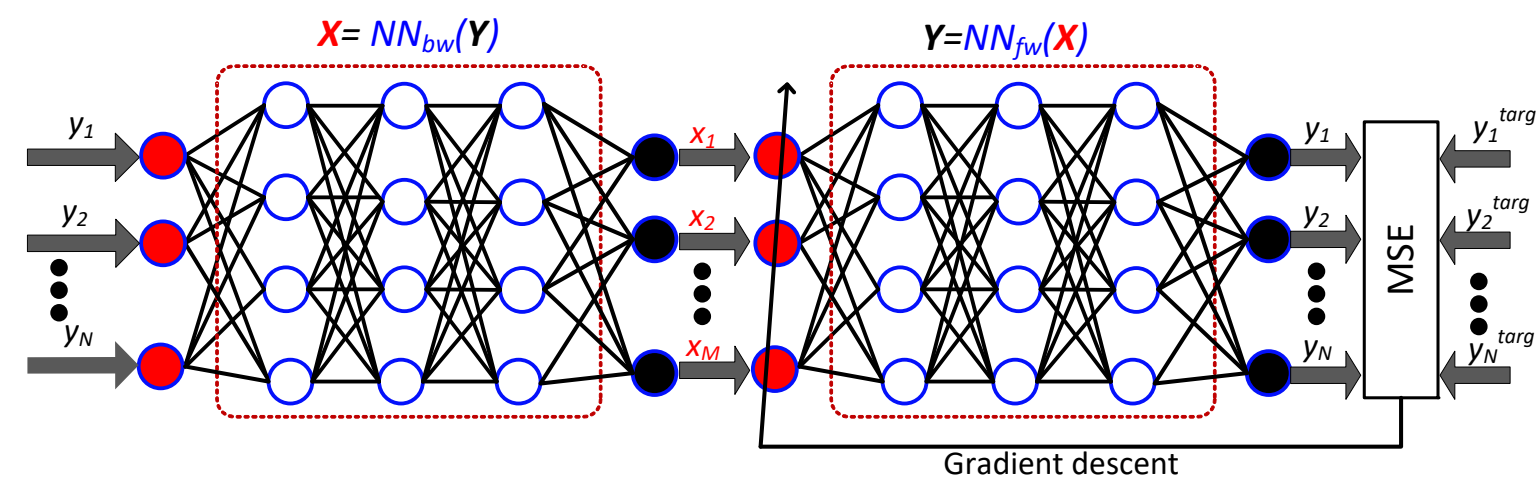

Fig. 1. Illustration of the machine learning based approach for the inverse system design [4].

desired gain profile. Moreover, we will show that Bayesian inference, an important ML technique, can enable accurate optical phase tracking yielding quantum-noise limited bit error rate (BER) performance.

\section{Inverse system design}

In the inverse system design, we are given a target output $\mathbf{Y}^{\text {targ }}$ and the objective is to determine the corresponding input $\mathbf{X}^{\text {targ }}$. The forward mapping is denoted by $\mathbf{Y}=f(\mathbf{X})$ and, to determine $\mathbf{X}^{\text {targ }}$, the inverse (backward) mapping function $f^{-1}(\cdot)$ needs to be determined such that $\mathbf{X}^{\text {targ }}=f^{-1}\left(\mathbf{Y}^{\text {targ }}\right)$. In many cases of interest, the forward mapping function $f(\cdot)$ is highly complex and described by a set of nonlinear differential or integral equations that must be solved numerically. Moreover, for some system, $f(\cdot)$, may even be unknown. Such cases may arise for the experimental implementations of optical communication systems. Therefore it is very challenging, and in some cases impossible, to obtain an expression for the gradient and apply standard least-squares optimization techniques to find $\mathbf{X}^{\text {targ }}$.

We have recently proposed a machine learning based framework, illustrated in Fig. 1, for the ISD [4]. The procedure is as following. First, a training data set containing the inputs, $\mathbf{X} \in \mathbb{R}^{N \times 1}$, and the outputs, $\mathbf{Y} \in \mathbb{R}^{M \times 1}$, of the system is generated: $\mathscr{D}^{K \times(N+M)}=\left\{\mathbf{Y}_{k}^{T}, \mathbf{X}_{k}^{T} \mid k=1, \ldots, K\right\}$, where $K$ is the size of the data set, $N$ is the number of inputs and $M$ is the number of outputs. In the simulation environment this is achieved by running the system model described by $f(\cdot)$. In the experimental environment this is achieved by exciting the system with a set of inputs and measuring the desired outputs. Given the data set $\mathscr{D}$ we can build a multi-layer neural network that learns the inverse mapping from the data, i.e. $\mathbf{X}=N N_{b w}(\mathbf{Y})=f^{-1}(\mathbf{Y})$. An ultra-fast prediction of $\mathbf{X}^{\text {targ }}$ given $\mathbf{Y}^{\text {targ }}$ can then be achieved as $\mathbf{X}^{\text {targ }}=N N_{b w}\left(\mathbf{Y}^{\text {targ }}\right)$ only contains matrix multiplications.

If the accuracy of $N N_{b w}(\cdot)$ is not satisfactory a gradient descent or some other optimization algorithms can be employed to perform fine-adjustment of the input $\mathbf{X}^{\text {targ }}$. The error is defined as the difference between the output $\mathbf{Y}=N N_{f w}\left(\mathbf{X}^{\text {targ }}\right)$ and the targeted output $\mathbf{Y}^{\text {targ }}$. This implies that a multi-layer neural network learning the forward mapping, $\mathbf{Y}=N N_{f w}(\mathbf{X})$ first needs to be built. Since $N N_{f w}(\cdot)$ contains matrix multiplications, the gradients can be computed and the optimization can be performed. This is illustrated in Fig. 1.

We have recently used the proposed framework for the pump power and wavelength allocation that would result in a desired arbitrary gain profile of the Raman amplifier operating in $\mathrm{C}$ and $\mathrm{C}+\mathrm{L}$ bands [4]. A low mean (0.46 and $0.35 \mathrm{~dB})$ and standard deviation $(0.20$ and $0.17 \mathrm{~dB})$ of the maximum error for numerical $(\mathrm{C}+\mathrm{L}-\mathrm{band})$ and experimental (C-band) results, respectively, when employing 4 pumps and $100 \mathrm{~km}$ span length was demonstrated.

\section{Optical phase tracking at the quantum limit}

To investigate the performance of the optical phase tracking at the quantum limit, the experimental setup consisting of two independent lasers and a quantum-noise limited balanced receiver (1.1 GHz $3 \mathrm{~dB}$ bandwidth) followed by an analogue-to-digital converter (10 GS/s) for beat signal digitization is employed. The binary phase shift keying (BPSK) data encoding is performed after the beat signal has been digitized. The post-modulation technique avoids non-idealities originating from optoelectronic components in the system which can induce an implementation penalty. Since it is not possible to distinguish if the source of penalty is the optical phase tracking algorithm or the component non-idealities, the post-modulation is justified - we are mainly interested in knowing the limits of the proposed optical phase tracking framework. For the optical phase noise tracking, we use a Bayesian inference framework that relies on the Unscented Kalman Filter (UKF) in combination with Monte Carlo Markov Chain 
(MCMC). The input signal power is varied from $32 \mathrm{pW}$ to $2.1 \mathrm{nW}(-75 \mathrm{dBm}$ to $-59 \mathrm{dBm})$, corresponding to SNR levels in the range from $-11 \mathrm{~dB}$ to $-7 \mathrm{~dB}$ in $1.1 \mathrm{GHz}$ bandwidth. In reference [10], we have shown that quantum noise limited BER performance is achievable using the employed phase noise tracking technique.

To investigate if the Bayesian inference framework for phase noise tracking can provide quantum noise limited performance, its variance needs to be compared to the theoretical quantum-limited variance for the heterodyne measurement. The quantum noise limited variance, $\sigma_{Q L}^{2}$, corresponds to the minimum obtainable mean square error $(M S E)$ for a given measurement setup. The MSE is defined as $\sigma_{O L}^{2} \equiv M S E=E\left[\left(\phi_{k}^{\text {true }}-\phi_{k}^{\text {est }}\right)^{2}\right]$, where $\phi_{k}^{\text {true }}$ is the true phase noise, $\phi_{k}^{e s t}$ is the estimated phase, $E[\cdot]$ is the expectation operator and $k$ is the discrete time index.

For a time-varying phase the quantum-limited variance for a heterodyne measurement employing statistically optimum phase estimation has been derived to be: $\sigma_{Q L}^{2}=1 / \sqrt{2 N_{\Delta v}}$ [11]. Here, $N_{\Delta v}=P_{s} / h f \Delta v$ is the average number of photons per coherence time (the laser linewidth $\Delta v$ is inversely proportional to the coherence time). It should be emphasized that the quantum limit calculated by $\sigma_{Q L}^{2}$ assumes a Wiener process model of laser phase noise. For this model, the corresponding frequency noise (FN) spectrum is flat, and its magnitude corresponds to the laser linewidth. However, the Wiener process model is just an approximation to a true laser phase noise. Typically the laser FN spectra is not flat and therefore a conversion of FN spectra to linewidth or direct measurement of laser linewidth is necessary. The direct measurement of laser linewidth requires radio frequency (RF) spectrum analyzers with very high resolution, especially if the laser linewidth is well below $1 \mathrm{kHz}$. Lasers used for quantum communication have low phase noise making the linewidth measurement using RF analyzers challenging. A solution is to make a conversion from FN spectra or phase noise ( $\mathrm{PN}$ ) spectra to linewidth as highly accurate large bandwidth phase noise measurement can be obtained using Bayesian filtering [10].

The challenge is then how to convert the FN or PN spectra to linewidth. Although several solutions in the literature have been proposed, all these solutions are approximate and their accuracy decreases for small linewidths. It is therefore difficult to compute the theoretically achievable quantum limited variance $\sigma_{Q L}^{2}$ and to benchmark various phase noise tracking schemes.

\section{Conclusion}

Next-generation optical communication systems will need to provide large data rates as well as a secure way of transmitting the information. This implies a coexistence of classical and quantum signals for which reconfigurable and artificial intelligence (AI) enabled digital coherent receivers may play an important role. In this paper, we have given some successful examples of how machine learning techniques can enable the design of $\mathrm{C}+\mathrm{L}-$ band Raman based optical amplifiers as well provide phase tracking schemes that enable quantum limited system performance.

\section{Acknowledgements}

This project has received funding from the European Union's Horizon 2020 research and innovation program under the Marie Skłodowska-Curie grant agreement No 754462, the European Research Council through the ERCCoG FRECOM project (grant agreement no. 771878), EU project CiViQ (grant agreement no. 820466), the Innovation Fund Denmark, Quantum Innov. Center and Center for Macroscopic Quantum States (bigQ, DNRF142) and BMBF Germany under 16KIS0490.

\section{References}

1. P. J.Winzer, et al., "Fiber-optic transmission and networking : the previous 20 and the next 20 years," Optics Express, vol. 26, no. 18, 2019

2. A. Napoli, et al., "Towards multiband optical systems," Proceedings of Optical Fibre Communication (OFC), paper Tu3E.1, 2018.

3. E. Diamanti, et al.,"Distributing secret keys with quantum continuous variables," Entropy, vol. 17, 2015

4. D. Zibar, et al., "Inverse system design using machine learning: a Raman amplifier case," Journal of Lightwave Technology, (in print), 2019

5. C. Hager, et al., "Revisiting Multi-Step Nonlinearity Compensation with Machine Learning," In Proceedings of European Conference on Optical Communication (ECOC), 2019

6. F. N. Khan, et al., “An Optical Communication's Perspective on Machine Learning and Its Applications,” Journal of Lightwave Technology, vol. 37, no. 2, 2019

7. B. Karanov, et al.,"End-to-End Deep Learning of Optical Fiber Communications," Journal of Light. Technology, vol. 36, no. 20, 2018

8. R. T. Jones, et al., "End-to-end Learning for GMI Optimized Geometric Constellation Shape," In Proceedings of European Conference on Optical Communication (ECOC), 2019

9. S. Kleis et al., "Improving the secret key rate of coherent quantum key distribution with Bayesian inference," Journal Lightwave Technology vol. 37, no. 3, 2019

10. D. Zibar et al., "Highly—sensitive phase and frequency noise measurement technique using Bayesian filtering," Photonics Technology Letters, (in print), 2019

11. T. A. Wheatley, et al.,"Adpative optical phase estimation using time-symmetric quantum smoothing," Physical Review Letters, 104, 2010 